\title{
A New Method of Image Reconstruction for PET Using a Combined Regularization Algorithm
}

\author{
Abdelwahhab Boudjelal ${ }^{1,2(\bowtie)}$, Abderrahim El Moataz ${ }^{1}$, and Zoubeida Messali ${ }^{2}$ \\ 1 Image Team, University of Caen Normandy and the ENSICAEN in the GREYC \\ Laboratory, 6 Boulevard Maréchal Juin, 14050 Caen Cedex, France \\ \{abdelwahhab.boudjelal, abderrahim.elmoataz\}@unicaen.fr \\ 2 Electrical Engineering Laboratory LGE M'sila University, M'sila, Algeria \\ messalizoubeida@gmail.com
}

\begin{abstract}
Positron emission tomography (PET), is a medical imaging technique that provides functional information about physiological processes. The goal of PET is to reconstruct the distribution of the radioisotopes in the body by measuring the emitted photons. The computer methods are designed to solve the inverse problem known as "image reconstruction from projections." In this paper, an iterative image reconstruction algorithm ART was regularized by combining Tikhonov and total variation regularizations. In the first step, combined regularization algorithm of total variation and Tikhonov regularization was applied to the image obtained by ART algorithm in each iteration for background noise removal with preserving edges. The quality measurements and visual inspections show a significant improvement in image quality compared to other algorithms.
\end{abstract}

Keywords: Positron emission tomography $\cdot$ ART algorithm $\cdot$ SART algorithm $\cdot$ Total variation $\cdot$ Tikhonov

\section{Introduction}

Positron emission tomography (PET) $[1,2]$ is a medical imaging modality that is used widely in clinical practice and scientific research as it provides quantitative and non-invasive information about biochemical and physiological processes in vivo. In PET [3], a small amount of a radioactive compound labeled with a radioisotope, called a radiotracer, is usually introduced into a patient's body through intravenous injection or inhalation, and then the spatial and sometimes also the temporal distribution of the radioisotope, the decay of which generates photons, is reconstructed from the photon measurements.

The emitted photons detected by the detectors are collected in set of projection data or sinogram. The aim of emission tomography is to reconstruct the spatial distribution of the radioisotope from the sinogram data by considering 
geometrical factors, physical effects, and noise properties. The image reconstruction problem belongs to the class of inverse problems [4].

Reconstruction algorithms proposed in the literature can be divided into two classes: analytical and iterative.

The use of iterative image reconstruction algorithms [5-10] can circumvent all these shortcomings. Iterative reconstructions have the advantages of incorporating corrections for image-degrading factors to handle an incomplete, noisy, and dynamic data set more efficiently than analytic reconstruction techniques. The most widely used iterative algorithms in emission tomography are the ML-EM (maximum-likelihood expectation maximization) algorithm and its accelerated version OSEM (Ordered Subset EM). The ML-EM method was introduced by Dempster et al. in 1977 [11] and first applied to PET by Shepp and Vardi [12]. The algebraic reconstruction Technique (ART) [13], considered as an important class of iterative approaches [14], assume that the cross-sectional section consists of a set of unknown, and then establishes algebraic equations for the unknown in terms of Measured projection data.

The regularization techniques are generally divided into a projection method and a penalty method [15]. In this paper, we use the penalty method techniques which are the Tikhonov regularization [16,17], TV method [18,19] (Fig.4). In this paper, we combine the ART algorithm TV + Tikhonov regularization techniques. The regularization of Tikhonov is the form of the L2-norm method of regularization on the data and the terms of regularization of the inverse problem.

\section{Materials and Methods}

\subsection{The PET Imaging Model}

The reconstruction from projections of PET images is a particularity of the general inverse problem of estimating the radioactive activity map related to a measurement $p$ by

$$
p_{i}=A_{i, j} x_{j}+n_{i}
$$

In the process of ECT imaging reconstruction, $x$ is the reconstructed image, $p$ is the measurement of projection data, $A$ is the system matrix whose component $A_{i, j}$ accounts for the probability of a photon emitted from pixel $j$ being recorded into bin $i$ and $n_{i}$ is the random and scatter events that add a bias to each detector.

The aim of reconstructing a PET scan image is to provide a direct solution of $x$ from the raw data collected during PET scanning $p$. However, PET data have an inherent stochastic character. There are uncertainties related to several aspects of PET physics, including the decay process, the effects of attenuation, the scattered coincidences and the additive random coincidences, $n$. To solve these problems, we propose a novel regularised ART algorithm for the image reconstruction combined with Tikhonov + TV regularization. 


\subsection{Algebraic Reconstruction Technique (ART)}

The ART is sequential method, i.e., each equation is treated at a time, since each equation is dependent on the previous. The equation of ART [13] is given by

$$
x_{j}^{(k+1)}=x_{j}^{(k)}+\alpha \frac{p_{i}-\sum_{n=1}^{N} A_{i, n} x_{n}^{(k+1)}}{\sum_{n=1}^{N} A_{i, n}^{2}} A_{i, j}
$$

where $x_{j}^{(k+1)}$ and $x_{j}^{(k)}$ are the current and the new estimates, respectively; $\sum_{n=1}^{N} A_{i, n} x_{n}^{(k+1)}$ is the sum weighted pixels along ray $i$; for the $k^{\text {th }}$ iteration; $p_{i}$ is the measured projection for the $i^{t h}$ ray, and $\alpha$ is the relaxation parameter.

\subsection{The Proposed Regularization Method Combining Tikhonov with Total Variation}

Given $p$ and $A$, the image $x^{0}$ is recovered from the model

$$
\min _{x} \sum_{i=1}^{n^{2}}\left\|D_{i} x\right\|+\frac{\mu}{2}\|A x-p\|_{2}^{2},
$$

where $D_{i} u \in R^{2}$ denotes the discrete gradient of $x$ at pixel $i$, and the sum $\sum\left\|D_{i} x\right\|$ is the discrete total variation (TV) of $x$.

At each pixel an auxiliary variable $\mathbf{w}_{i} \in R^{2}$ is introduced to transfer $D_{i} u$ out of the non-differentiable term $\|\cdot\|_{2}$ as follows [20].

$$
\min _{\mathbf{w}, x} \sum_{i}\left\|\mathbf{w}_{i}\right\|_{2}+\frac{\mu}{2}\|A x-p\|_{2}^{2}, \quad \text { s.t. } \quad \mathbf{w}_{i}=D_{i} x
$$

The simple quadratic penalty scheme was adopted, yielding the following approximation model of (4):

$$
\min _{\mathbf{w}, u} \mathcal{Q}_{\mathcal{A}}(u, \mathbf{w}, \beta)
$$

The main challenge of the paper is that solving the problem (3) is through solving a series of the combined Tikhonov and total variation regularized image models

$$
\min _{x=x_{1}+x_{2}} \operatorname{TV}\left(x_{1}\right)+\frac{\beta}{2} \operatorname{Tikhonov}\left(x_{2}\right)+\frac{\mu}{2}\|A x-p\|_{2}^{2},
$$

Therefore, the intermediate results are corresponding to different size of $\beta$ and the final solution is corresponding to a huge $\beta$ where the TV regularization dominates and the Tikhonov regularization is almost ignorable.

Let $x=x_{1}+x_{2}$ and $D_{i} x_{1}=\mathbf{w}$, then $D_{i} x_{2}=D_{i} x-\mathbf{w}_{i}$, where $\mathbf{w}$ is the auxiliary variable [20]. Equation (5) based on operator splitting and the quadratic penalty, can be rewritten as 


$$
\begin{aligned}
\min _{x_{1}, x_{2}} \sum_{i}\left\|D_{i} x_{1}\right\|_{2} & +\frac{\beta}{2} \sum_{i}\left\|D_{i} x_{2}\right\|_{2}^{2} \\
& +\frac{\mu}{2}\left\|A\left(x_{1}+x_{2}\right)-p\right\|_{2}^{2},
\end{aligned}
$$

Here we consider the recovered image $x$ as a sum of two components: a piecewise constant component $x_{1}$ and a smooth component $x_{2}$. Correspondingly, (5) is considered as a combination of Tikhonov regularization and total variation regularization.

The proposed algorithm combines ART algorithm with Tikhonov + TV. The algorithm involves the following: The ART algorithm can be regularized by adding a penalty term in the denominator:

$$
x_{j}^{(k+1)}=x_{j}^{(k)}+\alpha \frac{p_{i}-\sum_{n=1}^{N} A_{i, n} x_{n}^{(k+1)}}{\sum_{n=1}^{N} A_{i, n}^{2}+\beta \frac{\partial \operatorname{Tikhonov+TV}\left(x_{j}^{k}\right)}{\partial x_{j}^{k}}} A_{i, j}
$$

The overall process is implemented in two steps by combining iterative reconstruction algorithm ART with the Tikhonov+TV regularization (8).

\section{Performance Evaluation}

To evaluate the reconstructed results two criterions are calculated for the four implemented algorithms in addition to the visual quality of the resulting reconstructed images, in addition to the relative norm errors and the visual quality of the reconstructed image. The relative norm error of the resulting images [21] is used and defined as:

Root Mean Squared Error (RMSE) [22] is very commonly used and makes for an excellent general purpose error metric for numerical predictions.

$$
\mathrm{RMSE}=\sqrt{\frac{1}{M N} \sum_{i=1}^{M} \sum_{j=1}^{N}(x(i, j)-\hat{x}(i, j))^{2}}
$$

where $x(i, j)$ is the value of the pixel in the test image $\hat{x}(i, j)$ and the value of the pixel in the reconstructed image, $N$ is the total number of pixels. Greater $R M S E$, means that the resulting reconstructed image is closer to the test image. We used also The filled contour plot displays isolines of the reconstructed images and the plot of profiles of reconstructed images comparisons.

We shall use the following phantoms in this paper. In Fig. 4, we have the digital Moby phantom [23] was used to simulate the few-view projection data. One typical frame of the phantom is shown in Fig. 4 with size of $256 \times 256$ pixels.

\section{Results and Discussion}

In this section, we compared the reconstruction results of the conventional ART algorithm, the proposed ART+ Tikhonov+TV (ART-TTV) algorithm and 
Simultaneous algebraic reconstruction technique (SART) [24] for image reconstruction in emission tomography. Our first study involved comparing the noise magnitude in high and low count regions of results images. To do this we first examined variance as a function of position for images of phantoms reconstructed using three algorithms. To generate projection data, we simply add Poisson noise to each of the attenuated projections of the phantoms. The noisy projections are then used to reconstruct the 2D Phantoms (Fig. 1).

The resultant reconstructed images obtained from conventional ART, ARTTTV and SART algorithms with 30 iterations, are shown in Fig. 2. From this Figures, the visual quality of the reconstructed image of the phantom using the ART-TTV algorithm is comparable to the other methods. As compared to some other methods, the experimental algorithm preserves edges better. The effectiveness of noise removal for the test algorithm was comparable to that of SART method; however, the intensity in the ROI was appreciably higher in the latter. Compared to the other methods, the ART-TTV method generates a superior intensity profile while preserving the edges.

The resultant of quality measurements $(R M S E)$ of reconstructed images obtained from these algorithms by varying the number of iterations, are shown in Fig. 3. The later demonstrates that ART-TTV is providing better quality measurements than that of conventional ART and SART. The number of iterations is much required in order to enhance the image quality.

To better compare these differences Fig. 4 plots the 1D line profile is the horizontal line that crosses the image in the two ROIs. Noisy images in uniform

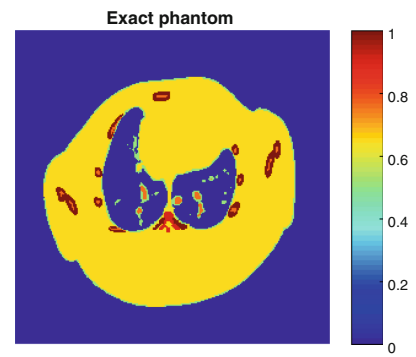

Fig. 1. Input image: Digital Moby phantom used in simulation study

Exact phantom

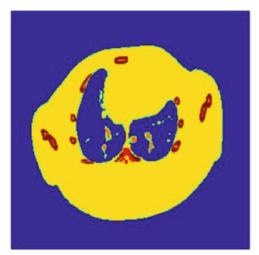

ART reconstruction

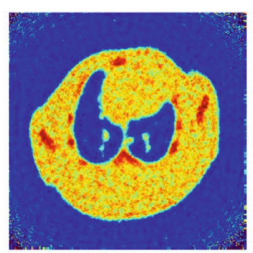

SART reconstruction

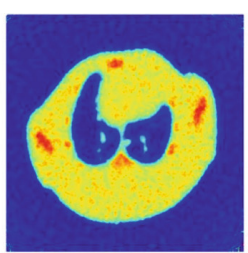

ART-TTV reconstruction

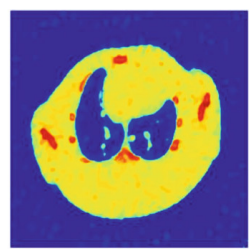

Fig. 2. Reconstructed images of the Moby phantom by different algorithms with 30 iterations. 


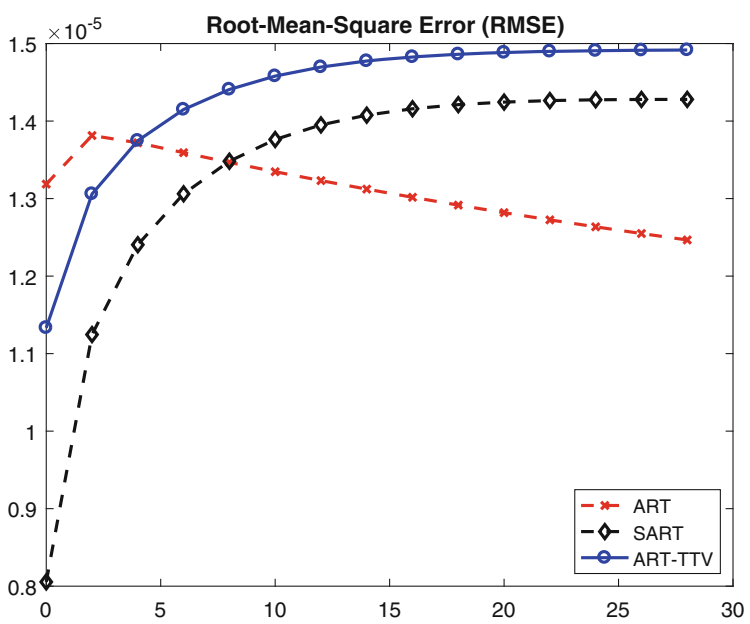

Fig. 3. RMSE vs iterations for ART, SART, and ART-TTV.

regions are shown as spikes as indicated by the SART; ART-TTV line profiles are closer to being noise free. The experimental method also nullifies aerial pixels by redistributing their values to pixels within the phantom. ART-TTV reconstructed the ideal profile more effectively than the other methods, and the image produced was a close approximation of the original phantom image.

All the visual-displays, the quality measurement and the line plots suggest that the proposed ART-TTV algorithm is preferable to the other algorithms. From all the above observations, it may be concluded that the proposed algorithm is performing better in comparison to conventional algorithms and provide a better reconstructed image.
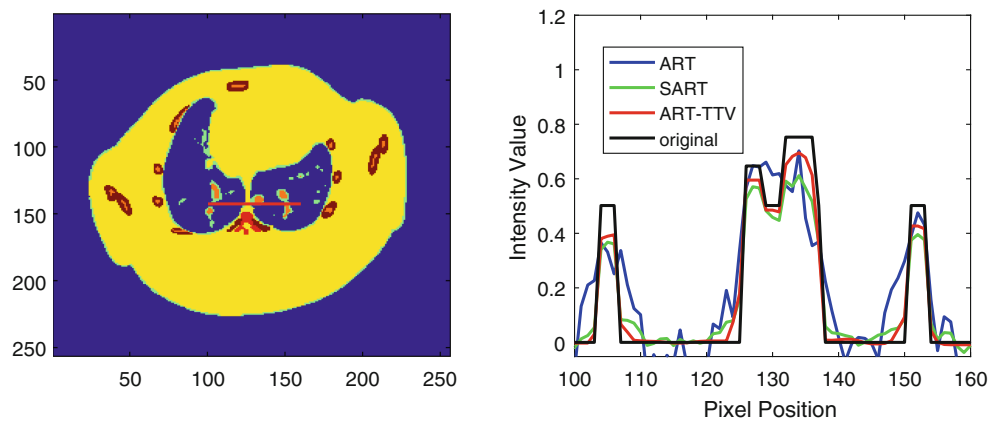

Fig. 4. Horizontal profiles through the images in Fig. 3 at the position of $y=145$. 


\section{Conclusion}

The new reconstruction algorithm has been presented for the reconstruction of projection data with insufficient iteration and data noisy. Under these conditions, the Digital Moby phantom simulation results verify that the proposed ARTTTV algorithm provides a clear improvement in reconstructed image quality and accuracy, compared with the other algorithms. The proposed algorithm produces an image, which is a close approximation of the original, improves its quality, and reduces noise and artifacts.

\section{References}

1. Phelps, M.E., Mazziotta, J.C., Schelbert, H.R.: Positron emission tomography. Los Alamos National Laboratory (1980)

2. Mazziotta, J.C., Schelbert, H.R.: Positron Emission Tomography and Autoradiography. Raven Press, New York (1985)

3. Turkington, T.G.: Introduction to PET instrumentation. J. Nucl. Med. Technol. 29(1), 4-11 (2001)

4. Bertero, M., Boccacci, P.: Introduction to Inverse Problems in Imaging. CRC Press, Boca Raton (1998)

5. Fessler, J.A.: Penalized weighted least-squares image reconstruction for positron emission tomography. IEEE Trans. Med. Imaging 13(2), 290-300 (1994)

6. Anastasio, M.A., Zhang, J., Xiaochuan Pan, Y., Zou, G.K., Wang, L.V.: Half-time image reconstruction in thermoacoustic tomography. IEEE Trans. Med. Imaging 24(2), 199-210 (2005)

7. Boudjelal, A., Attallah, B., Messali, Z.: Filtered-based expectation - maximization algorithm for emission computed tomography (ECT) image reconstruction. In: 2015 3rd International Conference on Control, Engineering \& Information Technology (CEIT), pp. 1-5. IEEE (2015)

8. Wernick, M.N., Aarsvold, J.N.: Emission Tomography: The Fundamentals of PET and SPECT. Academic Press, San Diego (2004)

9. Pan, X., Sidky, E.Y., Vannier, M.: Why do commercial CT scanners still employ traditional, filtered back-projection for image reconstruction? Inverse Prob. 25(12) (2009). https://doi.org/10.1088/0266-5611/25/12/123009

10. Boudjelal, A., Messali, Z., Elmoataz, A.: A novel kernel-based regularization technique for PET image reconstruction. Technologies 5(2), 37 (2017)

11. Dempster, A.P., Laird, N.M., Rubin, D.B.: Maximum likelihood from incomplete data via the EM algorithm. J. Roy. Stat. Soc.: Ser. B (Methodol.) 39(1), 1-22 (1977)

12. Shepp, L.A., Vardi, Y.: Maximum likelihood reconstruction for emission tomography. IEEE Trans. Med. Imaging 1(2), 113-122 (1982)

13. Gordon, R., Bender, R., Herman, G.T.: Algebraic reconstruction techniques (ART) for three-dimensional electron microscopy and X-ray photography. J. Theor. Biol. 29(3), 471IN1477-476IN2481 (1970)

14. Boudjelal, A., Elmoataz, A., Lozes, F., Messali, Z.: PDEs on graphs for image reconstruction on positron emission tomography. In: Mansouri, A., El Moataz, A., Nouboud, F., Mammass, D. (eds.) ICISP 2018. LNCS, vol. 10884, pp. 351-359. Springer, Cham (2018). https://doi.org/10.1007/978-3-319-94211-7_38 
15. Tehrani, J.N., Oh, T.I., Jin, C., Thiagalingam, A., McEwan, A.: Evaluation of different stimulation and measurement patterns based on internal electrode: application in cardiac impedance tomography. Comput. Biol. Med. 42(11), 1122-1132 (2012)

16. Tikhonov, A.N., Arsenin, V., John, F.: Solutions of Ill-Posed Problems, vol. 14. Winston, Washington, DC (1977)

17. Tikhonov, A.N., Goncharsky, A.V., Stepanov, V.V., Yagola, A.G.: Numerical Methods for the Solution of Ill-Posed Problems, vol. 328. Springer, Dordrecht (2013). https://doi.org/10.1007/978-94-015-8480-7

18. Rudin, L.I., Osher, S., Fatemi, E.: Nonlinear total variation based noise removal algorithms. Phys. D 60(1-4), 259-268 (1992)

19. Boudjelal, A., Messali, Z., Elmoataz, A., Attallah, B.: Improved simultaneous algebraic reconstruction technique algorithm for positron-emission tomography image reconstruction via minimizing the fast total variation. J. Med. Imaging Radiat. Sci. 48(4), 385-393 (2017)

20. Wang, Y.: FTVd is beyond fast total variation regularized deconvolution. arXiv preprint arXiv:1402.3869 (2014)

21. Kak, A.C., Slaney, M.: Principles of Computerized Tomographic Imaging. Society for Industrial and Applied Mathematics, Philadelphia (2001)

22. Chai, T., Draxler, R.R.: Root mean square error (RMSE) or mean absolute error (MAE)? Arguments against avoiding RMSE in the literature. Geosci. Model Dev. $\mathbf{7}(3), 1247-1250$ (2014)

23. Segars, W.P., Tsui, B.M.W., Frey, E.C., Johnson, G.A., Berr, S.S.: Development of a 4-D digital mouse phantom for molecular imaging research. Mol. Imaging Biol. 6(3), 149-159 (2004)

24. Andersen, A.H., Kak, A.C.: Simultaneous algebraic reconstruction technique (SART): a superior implementation of the ART algorithm. Ultrason. Imaging 6(1), 81-94 (1984) 